\title{
Rejection and Correction of the Fracture Mechanics Singularity Approach with its Associated Tree Modes of Crack Separation
}

\author{
T.A.C.M. van der Put ${ }^{1 *}$ \\ ${ }^{1}$ Technical University Delft, Netherlands \\ ${ }^{*}$ Corresponding author: T.A.C.M van der Put: vanderp@xs4all.nl
}

\section{OPEN ACCESS}

Citation: van der Put T.A.C.M. (2018) Rejection and Correction of the Fracture Mechanics Singularity Approach with its Associated Tree Modes of Crack Separation. Open Science Journal 3(2).

Received: $7^{\text {th }}$ February 2018

Accepted: $9^{\text {th }}$ April 2018

Published: $24^{\text {th }}$ May 2018

Copyright: (c) 2018 This is an open access article under the terms of the Creative Commons Attribution License, which permits unrestricted use, distribution, and reproduction in any medium, provided the original author and source are credited.

Funding: The author(s) received no specific funding for this work

Competing Interests: The author has declared that no competing interests exists.

\section{Abstract:}

Limit Analysis is an prescribed exact approach of Wood Science, what is shown to also apply for wood Fracture Mechanics. Knowledge of the gradual elastic to plastic behavior and of the imitation by non-linear elasticity (and J-integral) is shown to be not needed. The linear - full plastic limit approach delivers an elastic lower bound, up to this full plastic boundary, the fracture- or yield criterion, where ultimate load behavior is described, by virtual work approach and "flow" by the normality rule. This delivers the possibility to look at any equilibrium system, which satisfies compatibility and boundary conditions and nowhere exceeds this "flow" criterion and is verified by test data. Because the accepted singularity approach does not deliver a right mixed mode fracture criterion, it is necessary to make comparisons with other possible Airy stress functions. Therefore, the derivation of the accepted, general applied, elementary singularity solution with its 3 failure modes, is discussed and compared with new theory. This new limit analysis theory is based on an older, forgotten, Airy stress function, and shows e.g., by the new approach and application to wood, that there is no real difference between strength theory and fracture mechanics and between linear and non-linear theory. It delivers the, empirical verified, exact mixed mode failure criterion for wood; shows that stresses in the isotropic wood matrix also have to be regarded separately, to explain the, only by isotropy, possible, extremely high triaxial hydrostatic stress, and stress increase by the stress spreading effect. 
Therefore the stresses and strengths of the isotropic wood matrix are derived. The transformation to total stresses, including the reinforcement is shortly given for the empirical verification and for literature reference. Therefore, only the derivation of the necessary corrections of the singularity approach, for isotropic material, is regarded. This leads to a necessary rejection of the, tree failure modes, singularity approach of Irwin and of associate equations. By the splitting in 3 modes, there is no compatibility and no mixed mode fracture criterion. Instead there are tree, each excluding, Airy stress functions, thus 3, each excluding, compatibility equations. Necessary is one mixed mode solution for the total load. Then the solution also is known for separate acting (thus non zero) loading components. This is done in $\S 4$, necessarily in elliptic coordinates, to know failure, by the highest tangential, uniaxial tensile stress in the crack boundary. The tangential direction in polar coordinates is not tangential to the elliptic first expanded of the crack boundary. Therefore then not the right $K_{I I c}$ values are obtained. The expression in elliptic coordinates delivers (by the highest empirical correlation) the failure criterion of wood for every load combination. Transformation of this mixed mode solution to polar coordinates gives the corrected singularity method based on a mixed mode failure criterion and delivers also the definition of the stress intensity factor. This last also gives another interpretation of the Bazant curve, which is shown to be the initial mode I yield criterion.

Keywords: Fracture mechanics, Failure modes, Model of Irwin, Boundary value approach, Limit analysis approach

\section{Introduction}

A right, exact, failure criterion is an indispensable part of the strength calculation of design. Such, so called, mixed mode fracture criterion is lacking in the generally applied singularity approach. This is discussed in $\S 3$ and leads to the analysis of the inconsistencies of the separate failure modes description. It is shown in $\S 6$, that the right singularity equations follow from transformation to polar coordinates of the exact limit analysis equations of $\S 4$.

Based on extended strength research on clear wood and timber, [1][2][3][4] and fracture mechanics research of e.g. [5] and [6], it appeared that the failure criterion of strength theory is of the same form as the fracture mechanics critical crack extension criterion, (see $\S 4$ ). This shows strength theory to be the initially 
small crack fracture mechanics theory. This is empirically confirmed in $\S 2$, and thus the first discussed, because it is the starting point for the derivation of both, the singularity approach in $\S 3$ and the exact approach of $\S 4$.

It was derived in the past [1], that the tensor-polynomial equation should be regarded as a polynomial expansion of the real failure surface in stress space. Also is shown, in e.g. [4], that the third degree polynomial is identical to the real failure criterion and that the second degree part of the polynomial is identical to the orthotropic extension of the von Mises criterion for initial yield by microcrack extension. Further was shown that the third degree polynomial hardening terms of the criterion incorporate the $\S 4$ theoretical derived mixed mode I-II fracture equation, showing "hardening" to be based on the start of critical smallcrack extension, which is hindered by the reinforcement to follow the most critical direction.

The clear wood results, of [1], are also discussed together with the biaxial data for timber of [2], in e.g. [3], as result of a co-operative project. By that, all aspects of the failure criterion are known and are presented e.g. in [4] and empirically confirmed, e.g. in $\S 5$. Needed is still the here given discussion of the singularity approach with the necessary replacement for an exact approach.

A short introduction, to refer to, of the general applied calculation method is first needed.

Fracture by flat initial cracks is a two-dimensional problem and the boundary value approach should be based on the equilibrium equations and the compatibility condition in strains:

$2 \frac{\partial^{2} \gamma_{x y}}{\partial x \partial y}=\frac{\partial^{2} \varepsilon_{x}}{\partial y^{2}}+\frac{\partial^{2} \varepsilon_{y}}{\partial x^{2}}$

Substitution of the stress-strain relations in eq.(1.1), gives:

$(\chi+1)\left[\frac{\partial^{2} \sigma_{x}}{\partial y^{2}}+\frac{\partial^{2} \sigma_{y}}{\partial x^{2}}\right]+(\chi-3)\left[\frac{\partial^{2} \sigma_{x}}{\partial x^{2}}+\frac{\partial^{2} \sigma_{y}}{\partial y^{2}}\right]=8\left(\frac{\partial^{2} \tau_{x y}}{\partial x \partial y}\right)$

where $\chi=3-4 u$ for plane strain and $\chi=(3-v) /(1+u)$ for plane stress, and $v$ is Poisson's ratio. The stresses should satisfy the equilibrium equations which are after differentiation:

$\frac{\partial^{2} \tau_{x y}}{\partial x \partial y}=-\frac{\partial^{2} \sigma_{x}}{\partial x^{2}}-\rho \frac{\partial X}{\partial x}=-\frac{\partial^{2} \sigma_{y}}{\partial y^{2}}-\rho \frac{\partial Y}{\partial y}$,

where $\varrho$ is the density of the body, of the body force.

Substitution of eq.(1.3) in eq.(1.2) gives:

$(\chi+1)\left[\frac{\partial^{2}}{\partial y^{2}}+\frac{\partial^{2}}{\partial x^{2}}\right]\left(\sigma_{x}+\sigma_{y}\right)+4 \rho\left[\frac{\partial X}{\partial x}+\frac{\partial Y}{\partial y}\right]=0$

If body forces $\mathrm{X}$ and $\mathrm{Y}$ are derived from a potential $V$, so that:

$X=-\frac{\partial V}{\partial x}, \quad Y=-\frac{\partial V}{\partial y}$,

then eq.(1.4) becomes:

$\nabla^{2}\left(\sigma_{x}+\sigma_{y}\right)=0$. 
By the Airy stress function $U$ is for equilibrium:

$$
\sigma_{x}=\frac{\partial^{2} U}{\partial y^{2}}+\rho V ; \quad \sigma_{y}=\frac{\partial^{2} U}{\partial x^{2}}+\rho V ; \quad \tau_{x y}=-\frac{\partial^{2} U}{\partial x \partial y}
$$

and by substitution in eq.(1.6), the compatibility equation becomes:

$$
\nabla^{2}\left(\nabla^{2} U\right)=0
$$

or:

$$
\frac{\partial^{4} U}{\partial x^{4}}+2 \frac{\partial^{4} U}{\partial x^{2} \partial y^{2}}+\frac{\partial^{4} U}{\partial y^{4}}=0
$$

Important is that for compatibility of shear- and normal strains, eq.(1.2) or eq.(1.8) has to be applied for a solution. This is lacking for the total load in the singularity approach. In the following equations, the dash on top means, that it is the conjugate complex. Thus:

$$
\bar{\xi}=\xi-i \eta
$$

is the conjugate complex of: $\zeta=\xi+i \eta$. The derivative, always with respect to $z$, $(z=x+i y))$ is given by a slash. Thus, with aid of the Cauchy-Riemann equations, is:

$$
\zeta^{\prime}(z)=\frac{d \xi}{d z}=\frac{d \xi}{d x}+i \frac{d \eta}{d x}=\frac{d \xi}{d x}-i \frac{d \xi}{d y}
$$

The common textbook solution of the Airy stress function $U$ is in terms of two analytic functions $\phi(z)$ and $\chi(z)$, where $U$ is the real part of:

$$
\begin{aligned}
& \bar{z} \phi(z)+\chi(z) ., \\
& U=0.5[\bar{z} \phi(z)+z \bar{\phi}(z)+\chi(z)+\bar{\chi}(z)] \\
& \sigma_{x}=\partial^{2} U / \partial y^{2}=0.5\left[2 \phi^{\prime}(z)-\bar{z} \phi^{\prime \prime}(z)+2 \bar{\phi}^{\prime}(z)-z \bar{\phi}^{\prime \prime}(z)-\chi^{\prime \prime}(z)-\bar{\chi}^{\prime \prime}(z)\right] \\
& \sigma_{y}=\partial^{2} U / \partial x^{2}=0.5\left[2 \phi^{\prime}(z)+\bar{z} \phi^{\prime \prime}(z)+2 \bar{\phi}^{\prime}(z)+z \bar{\phi}^{\prime \prime}(z)+\chi^{\prime \prime}(z)+\bar{\chi}^{\prime}(z)\right] \\
& \tau_{x y}=-\partial^{2} U / \partial x \partial y=-0.5\left[i \bar{z} \phi^{\prime \prime}(z)-i z \bar{\phi}^{\prime \prime}(z)+i \chi^{\prime \prime}(z)-i \bar{\chi}^{\prime \prime}(z)\right]
\end{aligned}
$$

From these equations follows:

$$
\begin{aligned}
& \sigma_{y}-\sigma_{x}+2 i \tau_{x y}=\frac{\partial^{2} U}{\partial x^{2}}-\frac{\partial^{2} U}{\partial y^{2}}-2 i \frac{\partial^{2} U}{\partial x \partial y}=2\left[\bar{z} \phi^{\prime \prime}(z)+\chi^{\prime \prime}(z)\right] \\
& \sigma_{y}+\sigma_{x}=\frac{\partial^{2} U}{\partial x^{2}}+\frac{\partial^{2} U}{\partial y^{2}}=2\left[\phi^{\prime}(z)+\bar{\phi}^{\prime}(z)\right]=4 R\left[\phi^{\prime}(z)\right]
\end{aligned}
$$

Needed for $\S 4$ and $\S 6$ are transformations to curvilinear coordinates:

$$
z=\omega(\zeta)
$$

giving the function which connects point $\zeta=\xi+i \eta$ in the $\zeta$-plane to $z=x+i y$ in the $z$-plane.

Then follows from the equations (1.16) and (1.15):

$$
\begin{gathered}
\sigma_{\xi}+\sigma_{\eta}=\sigma_{x}+\sigma_{y}=2\left[\phi^{\prime}(z)+\bar{\phi}^{\prime}(z)\right] \\
\sigma_{\eta}-\sigma_{\xi}+2 i \tau_{\xi \eta}=\left(\sigma_{y}-\sigma_{x}+2 i \tau_{x y}\right) e^{2 i \delta}=2\left[\bar{z} \phi^{\prime \prime}(z)+\chi^{\prime}(z)\right] \cdot\left[\omega^{\prime}(\zeta) / \bar{\omega}^{\prime}(\zeta)\right] \\
\text { with: } \quad e^{2 i \delta}=\omega^{\prime}(\zeta) / \bar{\omega}^{\prime}(\xi)=\cos (2 \delta)+i \sin (2 \delta)
\end{gathered}
$$


Differentiation, is with respect to $z$, thus:

$$
\phi^{\prime}(z)=\frac{d \phi}{d \zeta} \frac{d \zeta}{d z}=\frac{1}{\omega^{\prime}(\zeta)} \frac{d \phi}{d \zeta}
$$

For transformation, according to eq.(1.17), to elliptic coordinates, is:

$z=x+i y=\omega(\xi)=c \cosh (\xi)=c \cosh (\xi+i \eta)=c \cosh (\xi) \cos (\eta) \rightarrow$

$d z / d \zeta=\omega^{\prime}(\xi)=c \sinh (\xi), \rightarrow x=c \cosh (\xi) \cos (\eta), \quad y=c \sinh (\xi) \sin (\eta)$

Thus, for a constant $\xi=\xi o$, the corresponding curve in the $\mathrm{x}, \mathrm{y}$ plane is the ellipse:

$$
\frac{x^{2}}{c^{2} \cosh ^{2}\left(\xi_{0}\right)}+\frac{y^{2}}{c^{2} \sinh ^{2}\left(\xi_{0}\right)}=1,
$$

with semi-axes: $a=c \cosh \left(\xi_{o}\right), \quad b=c \sinh \left(\xi_{o}\right) .$, . When $\eta=\eta_{o}$ is constant, the curves are hyperbolae, confocal with the ellipses (see Fig. 1):

$$
\frac{x^{2}}{c^{2} \cos ^{2}\left(\eta_{0}\right)}-\frac{y^{2}}{c \sin ^{2}\left(\eta_{0}\right)}=1
$$

The focus distance is $2 c$.

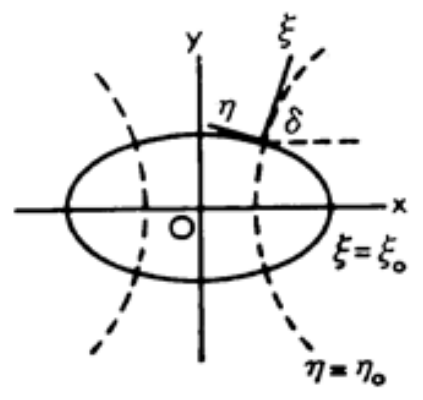

Figure 1 - Elliptic hole and elliptic coordinates [4]

This problem of the infinite region with an elliptic hole, loaded by an uniaxial stress $p$ inclined at $\beta$ to the crack plane, was solved, in elliptic coordinates, by Stevenson [7] by the following functions: $\phi(z)$, and $\chi^{\prime}(z)=\psi(z)$

$$
\begin{aligned}
& 4 \phi(z)=p c e^{2 \xi_{0}} \cos (2 \beta) \cosh (\zeta)+p c\left(1-e^{2 \xi_{0}+2 i \beta}\right) \sinh (\zeta) \\
& 4 \psi(z)=-p c\left[\cosh \left(2 \xi_{0}\right)-\cos (2 \beta)+e^{2 \xi_{0}} \sinh \left(2\left(\zeta-\xi_{0}-i \beta\right)\right)\right] \operatorname{cosech}(\zeta)
\end{aligned}
$$

Then for a flat elliptic crack, $\xi_{0}=0$, is:

$$
\sigma_{\xi}+\sigma_{\eta}=p \cos (2 \beta)+\alpha p[(1-\cos (2 \beta)) \sinh (2 \xi)-\sin (2 \beta) \sin (2 \eta)]
$$

$\sigma_{\xi}-\sigma_{\eta}=\alpha p \cosh (2 \xi) \cos (2(\eta-\beta))+\alpha^{2} p[(1-\cos (2 \beta))(\cos (2 \eta-1)) \sinh (2 \xi)+$ $-\cosh (2 \xi) \cos (2 \beta)+\cos (2(\eta-\beta))-\cosh (2 \xi) \sin (2 \beta) \sin (2 \eta)]$

$$
\begin{aligned}
& \tau_{\xi \eta}=0.5 p \alpha \sinh (2 \xi) \sin (2(\beta-\eta))+0.5 p \alpha^{2}[\sinh (2 \xi) \sin (2 \beta)(\cos (2 \eta)-1)+ \\
& \quad+(1-\cos (2 \beta))(\cosh (2 \xi)-1) \sin (2 \eta)
\end{aligned}
$$

where $\quad a=(\cosh (2 \xi)-\cos (2 \eta))^{--1}$

The transformation to polar coordinates is discussed in $\S 6$. 


\section{Unity of strength theory and small crack fracture mechanics}

The interpretation of the strength data-line of Fig. 2, of geometrically similar specimens of Bazant, is to regard, the inclined line to represent LEFM theory, the horizontal line to be the maximal stress, strength theory and the curved, connecting line, to follow nonlinear fracture theory. However, there is no difference between nonlinear and linear elastic (LEFM) fracture mechanics. For both the linear elastic - full plastic approach of limit analysis applies. When a specimen is loaded until flow, the following unloading and reloading is elastic. This elastic reloading until full plastic flow represents the limit analysis approach. The elastic boundary to the full-plastic zone exists as failure criterion, by a single curve in stress space as given by Fig. 2. In this figure of e.g. [8], is $d / d_{0}$, the ratio of specimen size to the fracture process zone size. But, because the line is the result of volume effect tests, the initial crack length is proportional to the testspecimen length. Thus, $d / d_{0}$, also can be regarded to be the ratio: initial open crack length, to the process zone size. Then, for small values of $\mathrm{d}$, this $d / d_{0}$, ratio also may represent the critical small crack density in a macro specimen (because $d$ also is the small crack interspace).

The curved line of Fig. 2, follows the equation:

$\ln \sigma=\ln \sigma_{0}-0.5 \ln \left(1+d / d_{0}\right)$

as result of a power law curve fitting. Eq.(2.1) therefore is:

$$
\begin{aligned}
& \ln \left(\frac{\sigma}{\sigma_{0}}\right)=\ln \left(\frac{d_{0}+d}{d_{0}}\right)^{-0.5}=\ln \left(\frac{d_{0}}{d_{0}+d}\right)^{0.5} \rightarrow \\
& \longrightarrow \sigma \sqrt{\pi\left(d_{0}+d\right)}=\sigma_{0} \sqrt{\pi d_{0}}=K_{c},
\end{aligned}
$$

in accordance with eq.(4.11). This confirms that the curve represents the stress intensity as ultimate state with $K_{\mathrm{c}}$ as critical stress intensity factor as should be for values of $d / d_{o}>>1$. For these higher values, the curved line approaches the drawn straight tangent line

$$
\ln \sigma=\ln \sigma_{0}-0.5 \cdot \ln \left(1+d / d_{0}\right) \approx \ln \sigma_{0}-0.5 \ln \left(d / d_{0}\right)
$$

with the necessary slope of the curve:

$$
\frac{\partial \ln \left(\sigma / \sigma_{0}\right)}{\partial \ln \left(d / d_{0}\right)} \approx-0.5
$$

as limit. The real slope however is

$$
: \frac{\partial \ln \sigma}{\partial \ln \left(d / d_{0}\right)}=\frac{\partial \ln \left(\sigma / \sigma_{0}\right)}{\left(d_{0} / d\right) \partial\left(d / d_{0}\right)}=\frac{d}{d_{0}} \frac{\partial\left(\ln \left(1+d / d_{0}\right)^{-0.5}\right)}{\partial\left(d / d_{0}\right)}=\frac{d}{d_{0}} \cdot \frac{-0.5}{1+d / d_{0}}=\frac{-0.5}{1+d_{0} / d}
$$

This slope is: -0.5 for $d>d_{o}$ and this slope is zero when $d=0$. This shows that for the whole curve LEFM applies and it is an indication that, at zero open crack dimensions, thus for: $d=0$, the clear wood ultimate strength theory still follows LEFM, because it applies also for the constant initial length $d_{o}$ (the constant fracture process zone length). After first yield drop, to half way unloading, maximal spreading is reached, and the strength theory further applies [4] for further unloading by crack extension. Similar to steel, where yield drop is due to dislocation multiplication and dislocation breakaway, applies for wood, that the start of yield drop is due to micro-crack multiplication (as fracture 
process zone $d_{o}$ ) and micro-crack propagation and merging (see [4]). The, in $\S 4$ derived, Wu-equation eq.(4.10), then reduces to eq.(4.9), expressed in stresses in stead of in stress intensities and, with $\tau_{u}=2 \sigma_{t}$ for isotropic

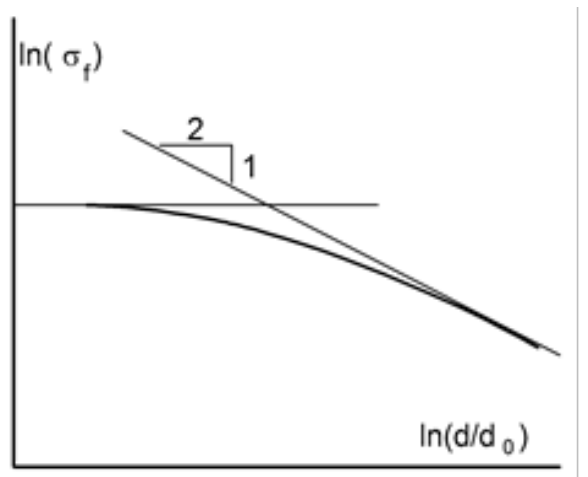

Figure 2. Limit LEFM behavior, [8], depending on the crack-length $d$ to process zone $d_{o}$ ratio showing no nonlinearity, thus a J-integral analog is not allowed.

matrix stresses, turns to the failure criterion of strength theory for clear wood eq.(2.7), when total stresses, in the reinforcement are accounted.

$$
1=\frac{\sigma_{y}}{\sigma_{t}}+\frac{\tau_{x y}^{2}}{\tau_{u}^{2}}
$$

It now follows that the general accepted application of the J-integral related models [9] to explain eq.(2.4) don't hold. This also is confirmed by the data given in [9]. For instance, Fig .3.27 of [9] of the critical crack tip opening displacements CTOD, of steel panels, show a scatter between 0.2 and $2 \mathrm{~mm}$. The Single Edge Notched Bend test, SE(B) shows a CTOD scatter (between 12,5 and 87.5 percentiles) of more than twice the median value. The same tendency follows for critical J integral values from SE(B) tests of Fig. 3.28 of [9]. Thus CTOD and Jintegral are not able to represent and explain fracture data, even not at real occurring plastic flow. To pass the lack of fit test of Table 1, it is necessary that, on first sight, mean data values are very close to theoretical curve, as applies for the in $\S 4$ discussed fracture criterion of wood.

\section{Rejection of the tree fracture modes singularity approach}

\section{Discussion of the derivation}

Stresses around a crack tip, at distance $r$ and direction $\vartheta$ are regarded to follow eq.(3.1) according to the now general accepted [10] fracture mechanics derivation [9], [11], [12]:

$$
\sigma_{i j}=\frac{K_{I}}{\sqrt{2 \pi r}} f_{i j}\left(\theta, s_{i j}\right)+\frac{K_{I I}}{\sqrt{2 \pi r}} g_{i j}\left(\theta, s_{i j}\right)+\frac{K_{I I I}}{\sqrt{2 \pi r}} h_{i j}\left(\theta, s_{i j}\right)
$$


where $\mathrm{K}$ is stress intensity and $s_{i j}$ are compliances This equation is based on the idea that a stress field can be divided according to 3 types of associated modes of deformation. The crack opening mode I, the sliding mode II and the pure torsional mode III. Discussed separately, in literature, is the solution, eq.(3.1), for $K_{I}=K_{I I}=0$, thus for loading by $K_{I I I}$ alone. Because a comparable failure interaction equation for the 3 modes is lacking, only eq.(3.1) for $K_{I}$ and $K_{I I}$, (with $K_{I I I}=0$ ) is regarded. As mentioned in $\S 1$, the solution of the biharmonic equation of the Airy stress function, which is necessarily in terms of two complex functions, and leads to the different terms of eq.(3.1), is based on a simple, most elementary, form in $r$ and $\vartheta$, containing 5 constants to adapt to boundary conditions and is, with: $z=r e^{i \vartheta}$ and $e^{i \vartheta}=\cos (\vartheta)+i \sin (\vartheta)$ :

$$
f_{1}=(\alpha+i \beta) z^{\lambda+1} \quad f_{2}=(\gamma+i \delta) z^{\lambda+1}
$$

The chosen symmetry conditions, of the displacements, for failure in the opening mode I, reduces this solution with two constants $\beta=\delta=0$ in eq.(3.2) and the two boundary conditions: eq.(3.3), then determine the constants: $\lambda=-0.5$ and $a=2 \gamma$ so that one constant: $\gamma$ remains, which is arbitrarily assumed to be KI. This means that arbitrarily variable $(c)^{1 / 2}$ is added, to form a new variable $\sigma_{\mathrm{ij}}(c)^{1 / 2}$, without any proof, what is not allowed. The result of the derivation was: $\sigma_{22}=2 \gamma r^{-1 / 2} f(\vartheta)$, where $\gamma$ is proportional to the loading stress $\mathrm{p}$ ad infinitum and $\sigma_{22}=\sigma_{t}$, the tensile strength, in the ultimate state. Thus: $\sigma_{\mathrm{t}}=2 \mathrm{p}(r)^{-1 / 2} f(0)$ determines the critical value of $p$. The derivation thus is a common derivation of strength theory and not of fracture mechanics, because the stress boundary conditions at the crack boundary are not satisfied. Thus the strength at the crack boundary is not regarded (as done in the right approach of $\S 4$ and $\S 6$ ). The argumentation that adding factor $c^{1 / 2}$ follows from dimensional analysis, only confirms that the strength at the crack boundary $2 c$ has to be regarded because the same dimensional argument does not apply for any other identical analysis of strength theory outside the crack.

The same procedure, as followed for mode I loading, is followed for mode II. Now $\alpha=\gamma=0$ by chosen anti-symmetry displacement conditions and because $\lambda=$ -0.5 , and $\beta$ and $\delta$ are related by the value of $K_{\text {IIc }}$ for the only present stress $\sigma_{12}$ at $\vartheta=0$, all constants are known. However, by this choice of separate failure modes, eq.(3.1) is not general valid in the ultimate state. Because the biharmonic Airy stress function, which is the strain compatibility equation, expressed in stresses, eq.(1.1), is different for symmetric and anti-symmetric loading, there is no compatibility for the total load sum according to eq.(3.1) and there is a mutual exclusion of the terms of eq.(3.1). The first term in $K_{I}$ only applies when $K_{I I}=$ $K_{I I I}=0$ and the second term in $K_{I I}$ only applies when $K_{I}=K_{I I I}=0$. Eq.(3.1) thus is in fact a meaningless stress superposition, of the results of the each excluding solutions for alone mode I, alone mode II and alone mode III. Needed for compatibility of shear and normal strains, is one Airy stress function (eq.(1.2) or eq.(1.8)), for the total load $K_{I}$ plus $K_{I I} \quad\left(K_{I I I}=0\right)$ of eq.(3.1). This single compatibility for the total load, is applied in $\S 4$ and $\S 6$, as correction of the, not compatible tree modes singularity approach. This leads to a real linear elastic mixed mode fracture criterion as general solution.

Because for the derivation of eq.(3.1), the crack is not regarded to be the limiting case of a flat ellipse, it should have been defined by: $-c<x<c$ causing two singularities at two end points: $x= \pm c$. By boundary condition eq.(3.3), is in fact half an infinite crack length regarded. Point $\vartheta= \pm \pi$, at $r=2 c$ (the crack 
length) is the opposite crack tip thus also a point of the crack boundary and thus is not stress free.

The, in [11], [12], chosen stress boundary definition: $K_{I}=\lim \left(\sigma_{22}(2 \pi r)^{1 / 2}\right)_{r \rightarrow 0}$ is arbitrary and is trivial, because the product $\left(\sigma_{22}(2 \pi r)^{1 / 2}\right)$ is independent of $r$. The right limit, where $\sigma_{22}=\sigma_{\mathrm{t}}$, the tensile strength, and $r=r_{0}$ of the crack boundary, is $K_{I c}=p(\pi c)^{1 / 2}$, as derived in $\S 4$. The aim of the chosen boundary condition, eq.(3.3), is stated in [11], to define a stress free crack surface. This is not right because this surface contains the highest tensile stresses near the crack tip, from where fracture starts. Therefore this condition:

$$
\sigma_{22}(\theta= \pm \pi)=\sigma_{12}(\theta= \pm \pi)=0
$$

has to be regarded to determine the stress free center of the crack. According to the exact approach of $\S 4$, this condition, eq.(3.3), is automatically fulfilled due to the stress free center of the flat elliptical crack. This also follows from the corrected singularity approach of $\S 6$, by applying eq.(3.3), when looking at these stresses at $\vartheta= \pm \pi$. As mentioned, conditions eq.(3.3), determine 2 constants of the specific chosen Airy stress function. For a non-trivial solution, then is necessary, that: $\lambda=-1 / 2, \mathrm{n} / 2$ with $\mathrm{n}=0,1,2, \ldots$ and the derived stress is:

$$
\sigma_{i j}=2(\lambda+1) r^{\lambda} \cdot f_{i j}\left(\theta_{5} s_{i j}\right) \quad \ldots . .=r^{-1 / 2} f_{i j}\left(\theta, s_{i j}\right)+\ldots . .=r^{-1 / 2} f_{i j}\left(\theta, s_{i j}\right)
$$

Thus, only one term, the first term with $r^{-1 / 2}$, applies, satisfying the boundary condition of a zero crack tip stress influence at infinitum. Thus the stated row solution with positive values of $\lambda$ does not exist. This also applies for the constant $(\lambda=0, \mathrm{n}=0)$, positive or negative term, what means that the $\mathrm{T}$ stress [9] of fracture mechanics of metals does not exist and by that, also not the Q-parameter, and other related variables.

In $\S 2$ and $\S 4$ is shown that, the already at the time of Griffith applied, maximal tensile stress failure criterion has to be used for extension of micro cracks at the fracture process zone.

This so called "maximum tangential stress" criterion is also applied for the derivation of eq.(3.1). However, the tangential direction in polar coordinates is not tangential to the elliptic crack boundary. Therefore not the right results are obtained. For instance: $K_{I I c}<K_{I c}$ is found, while normally $K_{I I c}>>K_{I c}$. (because $K_{I I c} \approx 2$ to 10 times $K_{I c}$ for materials, see $\S 4$ ). Because there always is tensile failure in the opening mode, shear sliding thus is due to elastic unloading after tensile failure in the opening mode, as confirmed by test specimens, showing no shear failure. Thus assumed anti-symmetry conditions don't apply at failure. Application of this maximal tangential tensile stress condition: $\partial \sigma_{\mathrm{tt}} / \partial \vartheta=0$, $\partial^{2} \sigma_{\mathrm{tt}} / \partial \vartheta^{2}<0$ on the components of eq.(3.1) leads to [11], [12]:

$$
K_{I} \sin (\theta)+K_{I I}(3 \cos (\theta)-1)=0
$$

This suggests, that eq.(3.5) is the mixed mode fracture criterion. However, there is no compatibility for combined loading, due to the, per load type, different applied Airy stress functions. Therefore, in [11], eq.(3.5) is split again into the separate two loading cases to determine the tensile strength for each case:

$$
K_{I} \sin (\theta)=0, \rightarrow \sin \left(\theta_{c}\right)=0, \rightarrow \theta_{c}=0
$$

for mode I loading alone, when $K_{I I}=0$, and:

$$
K_{I I}(3 \cos (\theta)-1)=0
$$


Or: $\cos (\vartheta)=1 / 3$ for mode II loading alone, when $K_{I}=0$, giving: $\theta_{c}= \pm \arccos (1 / 3)= \pm 70.6^{0}$

of which the negative angle $\vartheta_{\mathrm{c}}=-70.6^{\mathrm{o}}$ is determining.

According to the derivation of eq.(3.1) is this tangential stress:

$\sigma_{t t}=\frac{K_{I}}{\sqrt{2 \pi r}}\left(\frac{3}{4} \cos \left(\frac{\theta}{2}\right)+\frac{1}{4} \cos \left(\frac{3 \theta}{2}\right)\right)+\frac{K_{I I}}{\sqrt{2 \pi r}}\left(-\frac{3}{4} \sin \left(\frac{\theta}{2}\right)-\frac{3}{4} \sin \left(\frac{3 \theta}{2}\right)\right)$

and the tensile strengths follow from the critical $\vartheta_{\mathrm{c}}$ values of eq.(3.6) and (3.7).

Then for $K_{I I}=0$ and $\vartheta_{\mathrm{c}}=0$, the tensile strength is:

$$
\sigma_{t t u}=\frac{K_{I c}}{\sqrt{2 \pi r_{0}}}
$$

However, mathematically, this also applies for any value of $K_{I I}$ because $\mathrm{g}_{\mathrm{ij}}(\vartheta=0)$ in eq.(3.1). This is known to be untrue.

For a critical mode II load, when $K_{I}=0$, is, for failure by the tensile strength of eq.(3.9):

$$
\sigma_{t t u}=\frac{K_{I c}}{\sqrt{2 \pi r_{0}}}=\frac{\sqrt{4 / 3} \cdot K_{I I c}}{\sqrt{2 \pi r_{0}}} \rightarrow K_{I I c}=\sqrt{3 / 4} \cdot K_{I c} \approx 0.87 K_{I c}
$$

This is not a right outcome. The exact solution of $\S 4$ shows $K_{I I c}=2 K_{I c}$, as higher, thus more probable, lower bound, for isotropic material. Thus eq.(3.1) does not represent, in the ultimate state, the mixed mode failure criterion.

To investigate, whether eq.(3.1), as sum of 2 special, opposite, solutions of eq.(3.1), may represent, in the ultimate state, a not compatible failure state, eq.(3.5) has to be solved as follows:

Regarding a right angled triangle with longest side of 1 and shortest side of a, then, the third side is $\left(1-a^{2}\right)^{1 / 2}$ and $\sin (\vartheta)=a$, thus $\cos (\vartheta)=\left(1-a^{2}\right)^{1 / 2}$. Then eq.(3.5) becomes:

$$
\begin{aligned}
& K_{I} \sin (\theta)+K_{I I}(3 \cos (\theta)-1)=K_{I} a+K_{I I}\left(3 \sqrt{1-a^{2}}-1\right)=0 \rightarrow \\
& a^{2}\left(9+k_{I}^{2} / k_{I I}^{2}\right)-2 a \cdot k_{I} / k_{I I}-8=0 \\
& \rightarrow a=\frac{K_{I} / K_{I I}}{9+\left(K_{I} / K_{I I}\right)^{2}} \pm \sqrt{\left(\frac{K_{I} / K_{I I}}{9+\left(K_{I} / K_{I I}\right)^{2}}\right)^{2}+\frac{8}{9+\left(K_{I} / K_{I I}\right)^{2}}}=\sin \left(\theta_{c}\right)
\end{aligned}
$$

Thus depending on loading ratio $K_{I} / K_{I I}$, the critical value $\vartheta_{\mathrm{c}}$ is found, which has to be substituted in eq.(3.8). Because in eq.(3.12), the term: $8 /\left\{9+\left(K_{I} /\right.\right.$ $\left.\left.K_{I I}\right)^{2}\right\}$ strongly dominates, is the dependence on $K_{I} / K_{I I}$, negligible, especially for wood where $K_{I c} / K_{I I c}=1 / 7$ in the given example of $\S 5$, giving: $8 /(9+0.02)$ $=0.887 \approx 8 / 9=0.889$, thus independent of $K_{I} / K_{I I}$. Thus the sum of 2 each excluding solutions exclude together all possible solutions. This excludes eq.(3.1) again to be in the ultimate state a lower bound, mixed mode failure criterion. However, normally the lower bound, $K_{I I c} \approx K_{I c}$ would be chosen, according to eq.(3.9) and eq.(3.10). Then, the loading, of both $K_{I} \approx K_{I I}$ to the ultimate state, is a worst loading case. Thus for $K_{I}=K_{I I}$ is:

$$
a=0.1 \pm \sqrt{0.01+0.8}=0.1 \pm 0.9
$$


The solution: $\mathrm{a}=\sin (\vartheta)=1=\sin (\pi / 2)$ delivers a negative strength by eq.(3.8). Thus only negative values of $\vartheta$ and $\sin (\vartheta)$ apply, giving a maximal value of eq.(3.8), for: , $a=\sin (\theta)=-0.8=\sin (-0.9273) \rightarrow \theta_{c}=-0.927\left(=53^{0}\right)$

Substitution in eq.(3.8) then gives

$$
K_{I c}=K_{I}(0.716)+K_{I I}(1.0735)=K_{I}(1.789)=K_{I I}(1.789) \rightarrow K_{I}=K_{I I}=0.559 K_{I c}
$$

Thus at a loading $K_{I}=0.559 K_{I c}$, is $K_{I I}=0.559 K_{I c}$. This is close to, but higher than $0.5 K_{1 \mathrm{c}}$, so that correction by linear interpolation is possible. Where eq.(3.9) shows that for mode I alone applies: $K_{1} / K_{I c} \leq 1$, then for mode II alone, $K_{1 I} / K_{I I c} \leq 1$ should apply as correction, providing by interpolation the wanted linear (in $K_{1}$ and $K_{I I}$ ) failure relation like eq.(3.1):

$$
K_{I} / K_{I c}+K_{I I} / K_{I I c}=1
$$

This also applies for higher, real occurring values, of $K_{I I c}$, as is derived in [4], and may explain also, the empirical success of applying eq.(3.15).

\section{Discussion of SED-like fracture criteria}

An other applied criterion, to predict crack extension, is the minimum strain energy density (SED) criterion, which only applies for an elementary Airy stress function solution, which delivers stresses in $1 / \sqrt{ } r$. It could be expected that a SED-like criterion applies for brittle materials. Structural materials, however, show a higher order fracture energy, showing local "plasticity" to be determining. Therefore the same derivation, as done for the SED-equation, is possible, and more probable based on a critical distortional energy (CDE) criterion, (see [4] Appendix II) what leads, to the same equation as the SED-equation, with an identical form and number of terms, with only difference of numerical constants, thus leads to a comparable equation as the SED equation. It is thus probable that the critical CDE, and not the minimal SED- principle applies. The exact mixed mode failure criterion of $\S 4$, (the Wu-equation) which also directly can be derived, based on a critical distortional energy (see [4], § 5.2), is generally applicable and thus should replace a SED- or CDE- like criterion.

In the ultimate state, virtual work and virtual displacements are determining and the elastic state then has no influence on this ultimate value. Thus, a first order expanded of the compliance (IAW virtual work approach) should be used in the ultimate state. The in [10], on page 15 and page 86, prescribed, complicated formula for the orthotropic compliance does not fit to the data thus should not be used. The measured elementary compliance is right for calculations in the main direction. Also an increased compliance value (as applied in the Building Codes to adapt to total deformation) is allowed for the "elastic-full plastic" ultimate state approach of limit analysis. For compliance differences, the elementary linear theory (e.g. beam theory) applies because the triangle bending stress diagram and parabolic shear stress diagram represent the first expanded of a row expansion of any real, linear or curved, bending stress and shear stress division. Only the first expanded should be accounted at virtual changes. It is wrong to account for lower order effects, thus, e.g. also is forbidden, to account for the lower order clamping effect difference at virtual crack extension of notched beams. 


\section{Exact Lower Bound solution of fracture of the isotropic wood matrix}

The elliptic crack can be seen as first expanded of any crack form. The mathematics of stresses around a flat elliptic hole, (giving by this crack form, the highest stresses, and thus the most probable lower bound solution), have been discussed by many authors. Since the twentieth, the time of Griffith, many specific solutions were obtained based on the Airy stress function. The mathematics is given in textbooks and in the referenced literature. Here the analysis in elliptical coordinates with the Airy stress function of [7] is followed.

For the description of the elliptic crack, it is obvious to use elliptic coordinates, e.g. for giving the needed stress boundary conditions along the whole crack boundary, and the right crack tangent direction, necessary for an exact solution.

As mentioned in $\S 1$, the mathematical solution of the biharmonic Airy stress function equation, eq.(1.8) is given in terms of analytic functions $\phi(z)$ and $\chi(\mathrm{z})$.. Most problems are solved by taking these functions as polynomials or power series in $\mathrm{z}$ or $\mathrm{z}^{-1}$, where: $\mathrm{z}=\mathrm{x}+$ iy. Eq.(3.1) is a special example of such solution.

For the elliptic hole $\xi=\xi_{o}$ with semi-axes: $a=c \cosh \left(\xi_{o}\right)$ and $b=c \sinh \left(\xi_{o}\right)$ in an infinite region with uniaxial stress $\mathrm{p}$ at infinity, inclined at $\beta$ to the major axis $\mathrm{Ox}$ of the ellipse, (see Fig. 3), the functions $\phi(\mathrm{z})$ and $\chi^{\prime}(\mathrm{z})=\psi(\mathrm{z})$ are according to [7]:

$$
\begin{aligned}
& 4 \phi(z)=p c e^{2 \xi_{0}} \cos (2 \beta) \cosh (\zeta)+p c\left(1-e^{2 \xi_{0}+2 i \beta}\right) \sinh (\zeta) \\
& 4 \psi(z)=-p c\left[\cosh \left(2 \xi_{0}\right)-\cos (2 \beta)+e^{2 \xi_{0}} \sinh \left(2\left(\zeta-\xi_{0}-i \beta\right)\right)\right] \operatorname{cosech}(\zeta)
\end{aligned}
$$

These equations (4.1) and (4.2) satisfy the required conditions at infinity and at the surface: $\xi=\xi_{0}$ of the elliptic hole. Using eq.(1.22), $d z / d \zeta=\omega^{\prime}(\zeta)$ $=c \sinh (\zeta)$, it follows that:

$$
4 \phi^{\prime}(z)=4 \frac{d \phi}{d \zeta} \frac{d \zeta}{d z}=p e^{2 \xi_{0}} \cos (2 \beta)+p\left(1-e^{2 \xi_{0}+2 i \beta}\right) \operatorname{coth}(\zeta)
$$

The tangential stress $\sigma \mathrm{t}$ at the crack boundary $\xi=\xi_{0}$ is simply: $\sigma_{t}=\sigma_{\eta}$ because there $\sigma_{\zeta}=0$, and using (4.3), and because:

$$
\begin{aligned}
& \sigma_{\xi}+\sigma_{\eta}=\sigma_{x}+\sigma_{y}=2\left[\phi^{\prime}(z)+\bar{\phi}^{\prime}(z)\right], \rightarrow \sigma_{t}=2\left[\phi^{\prime}\left(\xi_{0}+i \eta\right)+\phi^{\prime}\left(\xi_{0}-i \eta\right)\right]= \\
& =p e^{2 \xi_{0}} \cos (2 \beta)+0.5 p\left(1-e^{2 \xi_{0}+2 i \beta}\right) \operatorname{coth}\left(\xi_{0}+i \eta\right)+ \\
& 0.5 p\left(1-e^{2 \xi_{0}-2 i \beta}\right) \operatorname{coth}\left(\xi_{0}-i \eta\right)= \\
& =p e^{2 \xi_{0}} \cos (2 \beta)+p\left[\cosh \left(2 \xi_{0}\right)-\cos (2 \eta)\right]^{-1} \sinh \left(2 \xi_{0}\right)+ \\
& -p e^{2 \xi_{0}}\left[\cos (2 \beta) \sinh \left(2 \xi_{0}\right)+\sin (2 \beta) \sin (2 \eta)\right]\left[\cosh \left(2 \xi_{0}\right)-\cos (2 \eta)\right]^{-1}= \\
& =p \frac{\sinh \left(2 \xi_{0}\right)+\cos (2 \beta)-\exp \left(2 \xi_{0}\right) \cos (2(\beta-\eta))}{\cosh \left(2 \xi_{0}\right)-\cos (2 \eta)}
\end{aligned}
$$

This eq.(4.4) can be extended by superposition to two stresses at infinity: $p_{2}$ inclined at $\beta$ to $\mathrm{Ox}$ and $p_{1}$ at $\pi / 2+\beta$, making any loading combination $\left(\sigma_{\mathrm{y}} \tau_{\mathrm{xy}}\right)$ possible, according to:

$$
\sigma_{x}=p_{1} \sin ^{2}(\beta)+p_{2} \cos ^{2}(\beta), \sigma_{y}=p_{1} \cos ^{2}(\beta)+p_{2} \sin ^{2}(\beta), \quad \tau_{x y}=-0.5\left(p_{1}-p_{2}\right) \sin (2 \beta)
$$

giving: 


$$
\sigma_{t}=\frac{2 \sigma_{y} \sinh \left(2 \xi_{0}\right)+2 \tau_{x y}\left[\left(1+\sinh \left(2 \xi_{0}\right)\right) \cot (2 \beta)-\exp \left(2 \xi_{0}\right) \cos (2(\beta-\eta)) \operatorname{cosec}(2 \beta)\right]}{\cosh \left(2 \xi_{0}\right)-\cos (2 \eta)}
$$

For a flat crack, thus for small $\xi_{0}$ and $\eta$ this is:

$$
\sigma_{t}=\frac{2\left(\xi_{0} \sigma_{y}-\eta \tau_{x y}\right)}{\xi_{0}^{2}+\eta^{2}}
$$

The maximal tangential stress follows from $d \sigma_{t} / d \eta=0$. Thus:

$$
\tau_{x y}\left(\xi_{0}^{2}-\eta^{2}\right)+2 \xi_{0} \sigma_{y} \eta=0 \rightarrow \eta=\xi_{0}\left[\sigma_{y} \pm \sqrt{\left(\sigma_{y}^{2}+\tau_{x y}^{2}\right)}\right] / \tau_{x y}
$$

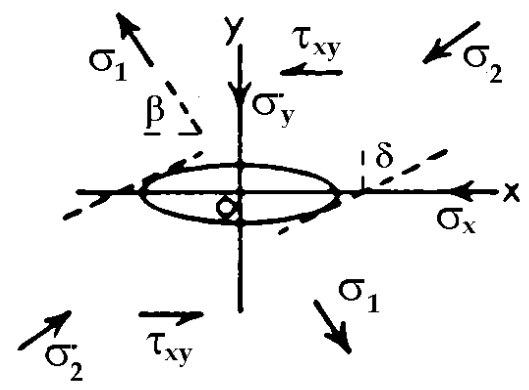

Figure 3. - Stresses in the notch plane Ox [4]

Substitutions in (4.6) gives:

$$
\xi_{0} \sigma_{t}=\sigma_{y} \pm \sqrt{\left(\sigma_{y}^{2}+\tau_{x y}^{2}\right)}
$$

This equation can be written:

$$
\begin{aligned}
& \left(\xi_{0} \sigma_{t}-\sigma_{y}\right)^{2}=\left( \pm \sqrt{\left(\sigma_{y}^{2}+\tau_{x y}^{2}\right)}\right)^{2}=\sigma_{y}^{2}+\tau_{x y}^{2}, \quad \rightarrow \\
& \frac{\tau_{x y}^{2}}{\left(\xi_{0} \sigma_{t}\right)^{2}}+\frac{\sigma_{y}}{\xi_{0} \sigma_{t} / 2}=1
\end{aligned}
$$

Transformation from elliptic to polar coordinates, by eq.(6.5) below, gives:

$$
\xi_{0}=\sqrt{2 r_{0} / c} \cdot \cos (\delta) \approx \sqrt{2 r_{0} / c}
$$

and substitution in eq.(4.9) gives:

$$
\frac{\sigma_{y} \sqrt{\pi c}}{\sigma_{t} \sqrt{\pi r_{0} / 2}}+\frac{\left(\tau_{x y} \sqrt{\pi c}\right)^{2}}{\left(\sigma_{t} \sqrt{2 \pi r_{0}}\right)^{2}}=\frac{K_{I}}{K_{I c}}+\frac{K_{I I}^{2}}{\left(K_{I I c}\right)^{2}}=1
$$

what should replace the ultimate value of eq.(3.1), while eq.(4.9) with constant $\xi_{o}$, for clear wood, gives the ultimate stress, strength criterion.

Eq.(4.10) is equal to the empirical Wu-equation. Wu noticed a jumping over fibres, what is identical to fracture propagation at a small value of $\delta$, which thus is neglected.

Eq.(4.10) further shows that fracture mechanics with constant stress intensity:

$$
K_{I c}=\sigma_{y} \sqrt{\pi c_{c}}=\sigma_{t} \sqrt{\pi r_{0} / 2}
$$

only applies when $r_{o}$ and $\sigma_{t}$, are constant. Thus $\sigma_{t}$, represents the cohesion strength at the crack tip craze and $r_{o}$ is the invariant radius of the constant dimensions of the, "fracture process zone" called, craze, at the crack tip. This zone clearly represents a kind of crazing with, as such, an invariant size, (which 
is regarded to be related to a material inhomogenities structure). As shown by eq.(6.14), hydrostatic tension occurs at the crack tip:

$$
\sigma_{r}=\sigma_{\theta}=p \sqrt{c / 2 r} \quad\left(\tau_{x y}=0\right)
$$

what also is equal to the third stress, due to confined contraction, $(\nu=0.5)$ what means, that these local stresses may become undetermined high, without failure in the isotropic wood matrix (lignin with branched hemicellulose). This need not apply for orthotropy, because then, for equal triaxial stresses, the strains are not equal and yield remains possible. However, for local compressional loading, the strong increase of strength, due to confined dilation, is generally known, see [13]. An "unconfined plasticity" calculation method (method of characteristic) based on stress spreading was already in the Dutch Code rules. The isotropic wood matrix therefore may sustain large stresses without yielding, because yield depends on a critical value of the distortional energy. High hydrostatic tension is, as well, possible in materials. Measured is e.g. 60 atmospheres for tension of water in a glass tube. Hydrostatic tension, made possible by a stress equalizing, stress spreading effect in wood thus is shown to be possible in the fracture process zone and explains the stress increase by the spreading effect for tension [4]. Crazing just occurs in regions of high hydrostatic tension, or in regions of very localized yielding, which leads to the formation of interpenetrating microvoids. At sufficient high tensile stress, the connections elongate and break, causing microvoids to grow and coalesce so that cracks begin to form. Crazing occurs in polymers, because that material is held together by a combination of weaker Van der Waals forces and stronger covalent bonds. Sufficient local stress overcomes the Van der Waals force, allowing a narrow gap. For wood, also the much stronger hydrogen bonds are involved. Once the slack is taken out of a backbone chain, covalent bonds, holding the chain together, hinder further widening of the gap. The gaps then are bridged by fibrils of the stretched backbone chain.

The process of craze growth, prior to cracking, absorbs fracture energy and effectively increases the fracture toughness of a polymer. The initial energy absorption per square meter in a craze region has been found to be up to several hundred times that of the un-crazed region, but quickly decreases and levels off. Crazes form at highly stressed regions, thus also at scratches, flaws, stress concentrations and molecular inhomogenities. Crazes generally propagate perpendicular to the applied tension (as cracks do). Crazing thus is typical for amorphous material of the isotropic wood matrix.

Because the high value of $\sigma \mathrm{t}$ and small ro are not known in eq.(4.10), but only the product $\sigma_{\mathrm{t}}\left(r_{o}\right)^{1 / 2}$, it is also possible to regard $\sigma_{\mathrm{t}}$ as flow stress and $r_{\mathrm{o}}$ as elastic-plastic boundary of limit analysis.

\section{Empirical verification of the mixed mode failure criterion}

For isotropic material eq.(4.10) predicts that $K_{I I c}=2 K_{I c}$. This is e.g. verified for Balsa wood, which is elastic orthotropic, but is extremely light, thus has a very low density of reinforcement, and thus is the isotropic matrix determining for the strength showing the isotropic strength behavior by $K_{I I c}=2 K_{I c}$ as 
verified by the data of $\mathrm{Wu}$ on Balsa (by $K_{I I c} \approx 140$ psi.in $^{0.5}$ and $K_{I c} \approx 60$ psi.in $^{0.5}$; (where $K_{I I c} \geq 2 K_{I c}$ by some hardening in the mode II test, and $K_{I c} \leq K_{I I c} / 2$ by early instability of the tension test).

The $\mathrm{Wu}$ - equation is generally applicable also when $\sigma_{y}$ is a compression stress as follows from the measurements. When the compression is high enough to close the small notches $\left(\sigma_{y, c l} \approx 2 G_{x y} \xi_{o}\right), \tau_{x y}$ has to be replaced by the effective shear stress:

$$
\tau_{\mathrm{xy}}^{*}=\tau_{\mathrm{xy}}+\mu\left(\sigma_{\mathrm{y}}-\sigma_{\mathrm{y}, \mathrm{cl}}\right)
$$

where $\mu$ is the friction coefficient, giving:

$$
1=\frac{\sigma_{\mathrm{y}, \mathrm{cl}}}{\xi_{0} \sigma_{\mathrm{t}} / 2}+\frac{\left(\tau_{\mathrm{xy}}^{*}\right)^{2}}{\xi_{0}^{2} \sigma_{\mathrm{t}}^{2}},
$$

what is fully able to explain fracture by compression perpendicular to the notch plane, [4].

The stress strain relations for orthotropic stresses in denser wood, can be given by:

$$
\varepsilon_{x}=c_{11} \sigma_{x}+c_{12} \sigma_{y} ; \quad \varepsilon_{y}=c_{12} \sigma_{x}+c_{22} \sigma_{y} ; \quad \gamma_{x y}=c_{66} \tau_{x y}
$$

Substitutions of eq.(5.2):

$$
\varepsilon_{x}=c_{11} \frac{\partial^{2} U}{\partial y^{2}}+c_{12} \frac{\partial^{2} U}{\partial x^{2}}, \ldots \ldots
$$

etc. in the compatibility condition:

$$
\frac{\partial^{2} \varepsilon_{x}}{\partial y^{2}}+\frac{\partial^{2} \varepsilon_{y}}{\partial x^{2}}=\frac{\partial^{2} \gamma_{x y}}{\partial x \partial y}
$$

gives:

$$
c_{22} \frac{\partial^{4} U}{\partial x^{4}}+\left(c_{66}+2 c_{12}\right) \frac{\partial^{4} U}{\partial x^{2} \partial y^{2}}+c_{11} \frac{\partial^{4} U}{\partial y^{4}}=0
$$

Wood acts as a reinforced material and can be treated to contain e.g. a shearreinforcement and a tensile reinforcement in the main direction. Then, for equilibrium of the matrix stresses (expressed in the total stresses) applies:

$$
\frac{\sigma_{x}}{n_{1}}=\frac{\partial^{2} U}{\partial y^{2}} ; \quad \sigma_{y}=\frac{\partial^{2} U}{\partial x^{2}} ; \quad \frac{\tau_{x y}}{n_{6}}=-\frac{\partial^{2} U}{\partial x \partial y},
$$

Inserted in the compatibility equation, eq.(5.3), this should give the isotropic Airy tress function. But the same compatibility should apply for matrix and reinforcement. Thus inserting the total stresses in eq.(5.3) should give a proportional result, given by eq.(5.6):

$$
c_{22} \frac{\partial^{4} U}{\partial x^{4}}+\left(n_{6} c_{66}+\left(1+n_{1}\right) c_{12}\right) \frac{\partial^{4} U}{\partial x^{2} \partial y^{2}}+n_{1} c_{11} \frac{\partial^{4} U}{\partial y^{4}}=0
$$

For the isotropic matrix thus is:

$$
n_{1} c_{11} / c_{22}=1 ; \quad\left(n_{6} c_{66}+\left(1+n_{1}\right) c_{12}\right) / c_{22}=2
$$

giving:

$$
\frac{\partial^{4} U}{\partial x^{4}}+2 \frac{\partial^{4} U}{\partial x^{2} \partial y^{2}}+\frac{\partial^{4} U}{\partial y^{4}}=\nabla^{2}\left(\nabla^{2} U\right)=0 \quad \rightarrow
$$




$$
n_{1}=\frac{c_{22}}{c_{11}}=\frac{E_{x}}{E_{y}} ; \quad n_{6}=\left(2-\frac{c_{12}}{c_{22}}-\frac{c_{12}}{c_{11}}\right) \cdot \frac{c_{22}}{c_{66}}=\left(2+v_{21}+v_{12}\right) \cdot \frac{G_{x y}}{E_{y}}
$$

This orthotropic-isotropic transformation of the Airy stress function in the elastic state of the reinforcement and the calculation method based on the stresses of the matrix makes it possible to use the isotropic solutions of $U$ to find the matrix stresses (which should not surmount the matrix strength). In this way eq.(4.10) becomes in total orthotropic stresses:

$$
1=\frac{\sigma_{\mathrm{y}}}{\xi_{0} \sigma_{\mathrm{t}} / 2}+\frac{\tau_{\mathrm{iso}}^{2}}{\xi_{0}^{2} \sigma_{\mathrm{t}}^{2}}=\frac{\sigma_{\mathrm{y}}}{\xi_{0} \sigma_{\mathrm{t}} / 2}+\frac{\tau_{\mathrm{ort}}^{2}}{\xi_{0}^{2} \sigma_{\mathrm{t}}^{2} \mathrm{n}_{6}^{2}}=\frac{\mathrm{K}_{\mathrm{I}}}{\mathrm{K}_{\mathrm{Ic}}}+\frac{\left(\mathrm{K}_{\mathrm{II}}\right)^{2}}{\left(\mathrm{~K}_{\text {IIc }}\right)^{2}}
$$

and it follows that:

$$
\begin{aligned}
& \frac{\mathrm{K}_{\text {IIc }}}{\mathrm{K}_{\text {Ic }}}=\frac{\xi_{0} \sigma_{\mathrm{t}} \mathrm{n}_{6}}{\xi_{0} \sigma_{\mathrm{t}} / 2}=2 \mathrm{n}_{6} \\
& 2 n_{6}=2\left(2+v_{21}+v_{12}\right) \cdot G_{x y} / E_{y}=
\end{aligned}
$$

$=2(2+0.57) / 0.67=7.7$ for Spruce and: $2(2+0.48) / 0.64=7.7$ for Douglas Fir in TL-direction, according to data of [10]. This is, in this chosen example, independent of the densities of respectively 0.37 and 0.50 at a moisture content of $12 \%$. Thus, for $\mathrm{K}_{\mathrm{Ic}} \approx 265 \mathrm{kN} / \mathrm{m}^{1.5}$, is $\mathrm{K}_{\mathrm{IIc}}=7.7 \times 265=2041 \mathrm{kN} / \mathrm{m}^{1.5}$ in TLdirection. In RL-direction this factor is 3.3 to 4.4 . Thus, when $\mathrm{K}_{\text {IIc }}$ is the same as in the TL-direction, the strength in RL-direction is predicted to be a factor 1.7 to 2.3 higher with respect to the TL-direction. This however applies at high crack velocities ("elastic" failure) and is also dependent on the site of the notch. At common loading rates a factor lower than $410 / 260=1.6$ is measured [10] and at still lower cracking speeds, this strength factor is expected to be about 1 when fracture is in the "isotropic" middle lamella. It then thus is independent of the TL and RL-direction according to the local stiffness and rigidity values. To know the mean influence, it is necessary to analyze fracture strength data dependent on the density and the elastic constants of $n_{6}$. From the rate dependency of the strength follows an influence of viscous and viscoelastic processes. This has to be analyzed by Deformation Kinetics [14].

Empirical verification of the above derived theory equation, eq.(5.8), which is a Coulomb equation, often called Wu-equation for wood, is not only obtained by [5], but also by tests of [6], done at the TL-system on eastern red spruce at normal climate conditions using different kinds of test specimens. The usual finite element simulations provided the geometric correction factors, and the stress intensity factors. The lack of fit test was performed on these data, at the for wood usual variability, assuming the five different, often suggested empirical

Table 1. - Lack of fit values for different failure criteria [6]. Statistical p-value

\begin{tabular}{|l|l|}
\hline Failure criterion & p-value \\
\hline$K_{I} / K_{I c}=1$ & 0.0001 \\
\hline$K_{I} / K_{I c}+K_{I I} / K_{I c}=1$ & 0.0001 \\
\hline$K_{I} / K_{I c}+\left(K_{I I} / K_{I I}\right)^{2}=1$ & 0.5629 \\
\hline$\left(K_{I} / K_{I c}\right)^{2}+K_{I I} / K_{I I}=1$ & 0.0784 \\
\hline$\left(K_{I} / K_{I c}\right)^{2}+\left(K_{I I} / K_{I C}\right)^{2}=1$ & 0.0001 \\
\hline
\end{tabular}


failure equations of Table 1. The statistical lack of fit values in the table show, that only the Wu-failure criterion, the third equation of Table 1 , cannot be rejected due to lack of fit. The Wu-equation is shown to fit also clear wood and timber strength data in [3] and [1], as expected from theory.

\section{Corrected Singularity equations, following from the exact solution}

For correction of eq.(3.1), which gives stresses near the crack tip in polar coordinates, it is sufficient to transform the exact equations from elliptical coordinates to Cartesian and polar coordinates. The stresses, outside the crack boundary follow from the solution of the Airy stress function of $\S 4$. A point near the crack tip of ellipse $\xi=\xi_{o}$ with coordinates:

$$
x=c \cosh (\xi) \cos (\eta), \quad y=c \sinh (\xi) \sin (\eta),
$$

with the focus $\mathrm{x}=\mathrm{c}$ of the ellipse as new origin with Cartesian coordinates $\mathrm{X}$, $\mathrm{Y}$, (see Fig. 4) is for small values of $\xi$ and $\eta$ :

$$
X=x-c=c\left(\xi^{2}-\eta^{2}\right) / 2, \quad Y=y=c \xi \eta
$$

what is in polar coordinates:

$$
r=\sqrt{X^{2}+Y^{2}} \quad X=r \cos (\theta), \quad Y=r \sin (\theta),
$$

From (6.2) follows:

$$
\xi^{2}+\eta^{2}=2\left(X^{2}+Y^{2}\right)^{1 / 2} / c=2 r / c
$$

And from (6.2) and (6.4):

$$
\begin{gathered}
\xi=(r / c)^{1 / 2}(1+\cos (\theta))^{1 / 2}=(r / c)^{1 / 2} \cos (\theta / 2) \\
\eta=(r / c)^{1 / 2}(1-\cos (\theta))^{1 / 2}=(r / c)^{1 / 2} \sin (\theta / 2)
\end{gathered}
$$

The quantity $\alpha$ of eq.(1.30) becomes:

$$
\alpha=(\cosh (2 \xi)-\cos (2 \eta))^{-1}=0.5\left(\xi^{2}+\eta^{2}\right)=c / 4 r
$$

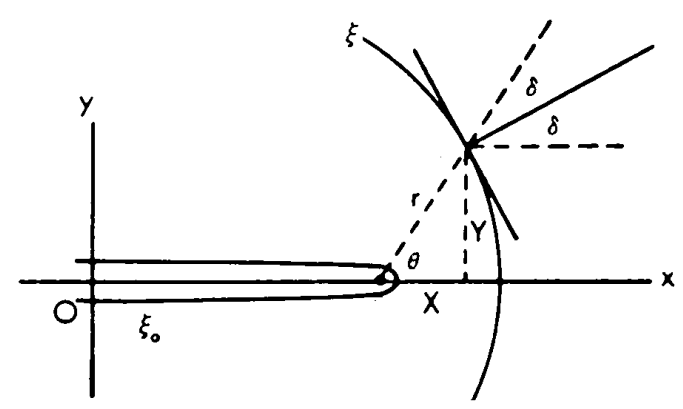

Figure 4.- Confocal coordinates of the elliptical crack [4]

The angle $\delta$ follows from

$$
\begin{aligned}
& e^{2 i \delta}=\omega^{\prime}(\zeta) / \bar{\omega}^{\prime}(\zeta)=(\sinh (\xi+i \eta)) /(\sinh (\xi-i \eta))=(\xi+i \eta) /(\xi-i \eta)=e^{i \theta} \\
& \text { or: } \delta=\vartheta / 2
\end{aligned}
$$


Substitution of this in equations (1.27) to (1.29) gives for the flat crack $\xi_{0}=0$ for loading by stress $p$ at infinity at an angle $\beta$ to the crack:

$$
\begin{aligned}
\sigma_{r} & +\sigma_{\theta}=\sigma_{\xi}+\sigma_{\eta}=p \cos (2 \beta)+\alpha p[(1-\cos (2 \beta)) \sinh (2 \xi)-\sin (2 \beta) \sin (2 \eta)]= \\
& =p \cos (2 \beta)+\alpha p[(1-\cos (2 \beta))(2 \xi)-(2 \eta) \sin (2 \beta)]= \\
& =p \cos (2 \beta)+p(c / 2 r)^{1 / 2}[(1-\cos (2 \beta)) \cos (\theta / 2)-\sin (2 \beta) \sin (\theta / 2)]
\end{aligned}
$$

The first term is constant and negligible with respect to the term in $(r)^{-1 / 2}$.

Proceeding in this way leads to:

$$
\begin{aligned}
& \left(8 r /\left(c p^{2}\right)\right)^{1 / 2} \sigma_{r}=\sin (\theta / 2) \cdot\left(1-3 \sin ^{2}(\theta / 2)\right) \sin (2 \beta)+2 \cos (\theta / 2) \cdot\left(1+\sin ^{2}(\theta / 2)\right) \sin ^{2}(\beta) \\
& \left(8 r /\left(c p^{2}\right)\right)^{1 / 2} \sigma_{\theta}=-3 \sin (\theta / 2) \cdot \cos ^{2}(\theta / 2) \cdot \sin (2 \beta)+2 \cos ^{2}(\theta / 2) \cdot \sin ^{2}(\beta) \\
& \left(8 r /\left(c p^{2}\right)\right)^{1 / 2} \tau_{r \theta}=\cos (\theta / 2) \cdot\left(3 \cos ^{2}(\theta / 2)-2\right) \cdot \sin (2 \beta)+2 \cos ^{2}(\theta / 2) \cdot \sin (\theta / 2) \cdot \sin ^{2}(\beta)
\end{aligned}
$$

For the common mode I test with collinear crack propagation is: $\beta=\pi / 2$ and $\vartheta=0$.

Then is: $\left(8 r /\left(c p^{2}\right)\right)^{1 / 2} \sigma_{r}=2$ and $\left(8 r /\left(c p^{2}\right)\right)^{1 / 2} \sigma_{\vartheta}=2, \quad$ and $\quad \tau_{r \vartheta}=0 . \quad$ Thus is:

$$
\sigma_{r}=\sigma_{\theta}=p \sqrt{c / 2 r}
$$

Showing, by the equal stresses, a local hydrostatic tension. This is discussed at eq.(4.12).

In general, for pure mode I, thus $\beta=\pi / 2$, follows:

$$
\begin{aligned}
& (2 r / c)^{1 / 2} \sigma_{r}=p \cos (\theta / 2) \cdot\left(1+\sin ^{2}(\theta / 2)\right) \\
& (2 r / c)^{1 / 2} \sigma_{\theta}=p \cos ^{3}(\theta / 2) \cdot \\
& (2 r / c)^{1 / 2} \tau_{r \theta}=p \cos ^{2}(\theta / 2) \sin (\theta / 2)
\end{aligned}
$$

For failure is:

$$
\partial \sigma_{\theta} / \partial \theta=0 . \rightarrow(2 r / c)^{1 / 2} \partial \sigma_{\theta} / \partial \theta=-(3 / 2) p \cos ^{2}(\theta / 2) \cdot \sin (\theta / 2)=0, \rightarrow \theta=0
$$

Thus for $\sigma_{\vartheta}=\sigma_{t}$, the tensile strength, is:

$$
p \sqrt{\pi c}=K_{I}=\sigma_{t} \sqrt{2 \pi r}=K_{I c}
$$

The loading case, for pure shear $S$, follows from eq.(6.11) to eq.(6.13) by superposition of $p=S$ at $\beta=\pi / 4$ with $p=-S$ at $\beta=3 \pi / 4$ giving:

$$
\begin{aligned}
& (2 r / c)^{1 / 2} \sigma_{r}=S \sin (\theta / 2) \cdot\left(1-3 \sin ^{2}(\theta / 2)\right) \\
& (2 r / c)^{1 / 2} \sigma_{\theta}=-3 S \sin (\theta / 2) \cos ^{2}(\theta / 2) \\
& (2 r / c)^{1 / 2} \tau_{r \theta}=S \cos (\theta / 2) \cdot\left(3 \cos ^{2}(\theta / 2)-2\right)
\end{aligned}
$$

For failure is: $\partial \sigma_{\vartheta} / \partial \vartheta=0 . \quad$ Thus:

$$
(2 r / c)^{1 / 2} \partial \sigma_{\theta} / \partial \theta=-(3 / 2) S\left\{\cos ^{3}(\theta / 2)-2 \sin ^{2}(\theta / 2) \cos (\theta / 2)\right\}=0, \rightarrow \tan (\theta / 2)=\sqrt{0.5}=0.707
$$

Thus:

$$
\begin{aligned}
& \sigma_{r} \approx \tau_{r \theta} \approx 0 \rightarrow \sqrt{2 \pi r} \sigma_{t}=-3 S \sqrt{\pi c}\left(0.577 \cdot 0.817^{2}\right)=-1.155 \cdot S \sqrt{\pi c} \rightarrow \\
& -S \sqrt{\pi c}=K_{I I}=\sqrt{2 \pi r} \sigma_{t} / 1.155=K_{I I c}=K_{I c} / 1.155 .
\end{aligned}
$$

The same was found by the derivation of eq.(3.1) in [11], [12], although an other Airy stress function was used, which however shows the same periodicity by the use of sinus and cosinus functions. The outcome difference with the exact 
solution of $\S 4$, where $K_{I I c}=2 K_{I c}$, thus is due to the fact that according to the singularity approach in polar coordinates, the direction $r \cdot d(\vartheta)$ is not tangential to the direction of the crack, but cut this crack boundary under an angle of $\vartheta / 2$ (see Fig. 4). Only for pure mode I, when $\vartheta=0$ the right tangential direction is given and thus the right outcome for $K_{I c}$. Thus the singularity approach, in polar coordinates gives, by regarding the wrong tangential direction of the crack, a far too low value of $K_{I I c}$, thus should be rejected and not be used. By the following the corrected equations will be given and the consequences of application of the wrong critical polar tangential stress criterion. Eq.(3.1) becomes, corrected for combined loading:

$$
\begin{gathered}
(2 r / c)^{1 / 2} \sigma_{r}=p \cos (\theta / 2) \cdot\left(1+\sin ^{2}(\theta / 2)\right)+S \sin (\theta / 2) \cdot\left(1-3 \sin ^{2}(\theta / 2)\right) \\
(2 r / c)^{1 / 2} \sigma_{\theta}=p \cos ^{3}(\theta / 2)-3 S \sin (\theta / 2) \cos ^{2}(\theta / 2) \\
(2 r / c)^{1 / 2} \tau_{r \theta}=p \cos ^{2}(\theta / 2) \sin (\theta / 2)+S \cos (\theta / 2) \cdot\left(3 \cos ^{2}(\theta / 2)-2\right)
\end{gathered}
$$

It follows that: : $\sigma_{\vartheta}=\tau_{r \vartheta}=0$ for $\vartheta= \pm \pi$.

It thus appears that condition eq.(3.3) is automatically fulfilled by the presence of the empty space in the centre of the flat elliptic crack. Thus this condition gives not condition of the absence of stress at the crack boundary, but gives the zero stresses in the centre of the crack.

The wrong critical tangential stress follows from $\partial \sigma_{\vartheta} / \partial \vartheta=0$ or:

$$
\begin{aligned}
& -3 p \sin (\theta / 2) \cos ^{2}(\theta / 2)+3 S\left(2 \cos (\theta / 2) \sin ^{2}(\theta / 2)-\cos ^{3}(\theta / 2)\right)=0 \\
& p \sin (\theta / 2) \cos (\theta / 2)+S\left(1-3 \sin ^{2}(\theta / 2)\right)=0 \rightarrow \\
& p \tan (\theta / 2)+S\left(1-2 \tan ^{2}(\theta / 2)\right)=0 \rightarrow \\
& \tan ^{2}(\theta / 2)-(p / 2 S) \tan (\theta / 2)-0.5=0 \\
& \quad \text { or finally: } \\
& \quad \tan (\theta / 2)=p / 4 S \pm \sqrt{(p / 4 S)^{2}+0.5}
\end{aligned}
$$

Thus for any load combination $p / S=K_{I} / K_{I I}$, the angle $\vartheta$ is known and by that all stresses are known. Substitution of eq.(6.27) in eq.(6.25) gives:

$$
\tau_{r \theta}=0 \text {. }
$$

Thus $\sigma \mathrm{r}$ and $\sigma \vartheta$ are principal stresses. Thus a local extreme is found.

Substitution of eq.(6.27) in eq.(6.23) gives:

$$
\sqrt{2 r / c} \cdot \sigma_{r}=p \cos (\theta / 2)
$$

Substitution of eq.(6.27) in eq.(6.24) gives, with $\sigma_{\vartheta}=\sigma_{t}$, the tensile strength: $\sqrt{2 r / c} \cdot \sigma_{t}=-S \cdot \cos ^{2}(\theta / 2) / \sin (\theta / 2)=|S| \cdot \cos ^{2}(\theta / 2) / \sin (\theta / 2)$

$\sigma_{\vartheta}$ is the highest stress when $\sigma_{\vartheta}>\sigma_{r}$, thus when: $p>|S|$. This is confirmed in the ultimate state, by eq.(6.22), for the regarded direction.

These equations, thus are based on stresses in an oblique direction of $\vartheta / 2$ to the elliptic crack boundary direction, which values are lower than the real critical, in plane values of $\S 4$.

The maximal tangential stress criterion thus does not apply for the equations in polar coordinates. Only for pure mode I, when $\vartheta=0$ the right tangential direction is given and thus the right outcome for $K_{I c}$. 


\section{Conclusions}

Limit Analysis is a prescribed exact approach of Wood Science, what is shown to apply also for wood Fracture Mechanics. The linear elastic -full plastic approach delivers simple lower bound equilibrium solutions, making judgement of results of other methods possible, e.g.

According to limit analysis, there is no difference between nonlinear and linear elastic (LEFM) fracture mechanics. For both the linear elastic - full plastic approach applies.

An other interpretation is given of the size effect curve of Bazant. Based on the mathematical expression, the curve shows to represent linear elastic fracture mechanics up to limit of strength theory for crazing at zero open crack length. The properties of the crazing strength appear to determine and define the strength behavior according to the stress intensity factor. Strength theory thus is identical to small crack fracture mechanics and thus basic for all fracture processes.

Stresses in the isotropic wood matrix have to be regarded separately, to explain the there, at crazes, possible, extremely high triaxial hydrostatic stress and the triaxiality by the stress spreading effect.

The derivation of the, only accepted, boundary value solution based on the separate 3 failure modes model of Irwin, is discussed what leads to rejection by the following remarks:

The general solution should, by definition, lead to a mixed mode fracture criterion. This is lacking in this singularity approach, what should lead to rejection of the method.

Condition eq.(3.3) does not mean that there is a stress free crack boundary, but indicates a zero stress at the centre of the crack. In the exact solution $\S 4$, and the corrected singularity solution $\S 6$, this condition applies automatically by the presence of the crack. The highest stresses occur at the crack boundary wherefore failure applies according to the maximal tangential tensile stress criterion. The Irwin solution thus does not satisfy the boundary and strength condition at the crack boundary. Therefore, the solution is an ultimate strength condition of strength theory, which is independent of the crack length 2c. This can not be corrected, as done, by simply putting variable $c^{1 / 2}$ before the stress in the strength solution and regard $\left(\sigma c^{1 / 2}\right)$ as new variable, without any derivation.

Stresses around a crack tip are regarded to follow eq.(3.1) of Irwin according to the now general accepted fracture mechanics, boundary value, derivation:

$$
\sigma_{i j}=\frac{K_{I}}{\sqrt{2 \pi r}} f_{i j}\left(\theta, s_{i j}\right)+\frac{K_{I I}}{\sqrt{2 \pi r}} g_{i j}\left(\theta, s_{i j}\right)+\frac{K_{I I I}}{\sqrt{2 \pi r}} h_{i j}\left(\theta, s_{i j}\right)
$$

The applied analysis shows that this is the sum of 3, each excluding, solutions in the 3 modes. The first term in $K_{I}$ applies for normal loading only and symmetric displacements, thus only when $K_{I I}=K_{I I I}=0$ and the second term in $K_{I I}$ applies for shear loading only when $K_{I}=K_{I I I}$, etc. Because the biharmonic Airy stress function, is the strain compatibility equation, expressed in stresses, eq.(1.1), is different for symmetry or anti-symmetry loading conditions, there is no compatibility for the total load sum according to eq.(3.1). The equation thus cannot be a linear elastic fracture mechanics equation. Needed for compatibility of shear and normal strains is one Airy stress function: eq.(1.2) or eq.(1.8), for 
the total load $K_{I}$ plus $K_{I I}$ plus $K_{I I I}$ of eq.(3.1). This is applied in $\S 4$ and $\S 6$, as correction of the, not compatible tree modes singularity approach.

The so called "maximum tangential stress" criterion is also applied for the derivation of eq.(3.1). However, the tangential direction in polar coordinates is not tangential to the elliptic first expanded of the crack boundary. Therefore not the right results are obtained but e.g. $K_{I I c}=0.87 K_{I c}$,.., while the exact solution gives $K_{I I c}=2 K_{I c}$. The same low value of $K_{I I c}=0.87 K_{I C}$ is found for the corrected singularity approach in polar coordinates, independent of the applied, mutual totally different, Airy stress functions. This is due to the same periodicity by the use of sinus and cosinus functions

This again leads to a necessary rejection of the separate 3 failure modes model of Irwin and of all solutions in polar coordinates, based on the maximal tangential stress failure criterion. The corrected, compatible singularity equations of $\S 6$ follow from transformation to polar coordinates of the exact limit analysis equations of $\S 4$. Of course, also for the corrected equations the applied polar maximal tangential stress criterion gives the wrong results.

The tangential direction of the flat elliptic crack should be regarded which represents the first expanded of any crack form and according to limit analysis, only application of first expanded terms are allowed. For this reason also beam theory has to be used for compliance differences according to the energy approach of fracture of beams. The linear bending stress diagram and parabolic shear stress diagram are the first expanded of the row expansion of any occurring bending and shear stress division.

It has to be concluded that the right results only can be obtained by a description by an elliptic crack and to use elliptic coordinates, e.g. for giving the needed stress boundary conditions along the whole crack boundary, and the right crack tangent direction, necessary for an exact solution, which is for a flat crack, thus for small $\xi_{o}$ and $\eta$ :

$$
\sigma_{t}=\frac{2\left(\xi_{0} \sigma_{y}-\eta \tau_{x y}\right)}{\xi_{0}^{2}+\eta^{2}}
$$

The maximal tangential stress follows from $d \sigma / d \eta=0$, leading to:

$$
\frac{\tau_{x y}^{2}}{\left(\xi_{0} \sigma_{t}\right)^{2}}+\frac{\sigma_{y}}{\xi_{0} \sigma_{t} / 2}=1
$$

For a constant $\xi_{o}$ this is the clear wood ultimate stress criterion of strength theory.

Transformation from elliptic to polar coordinates, by eq.(6.5), gives:

$$
\xi_{0}=\sqrt{2 r_{0} / c} \cdot \cos (\delta) \approx \sqrt{2 r_{0} / c}
$$

and substitution, in equation above, gives:

$$
\frac{\sigma_{y} \sqrt{\pi c}}{\sigma_{t} \sqrt{\pi r_{0} / 2}}+\frac{\left(\tau_{x y} \sqrt{\pi c}\right)^{2}}{\left(\sigma_{t} \sqrt{2 \pi r_{0}}\right)^{2}}=\frac{K_{I}}{K_{I c}}+\frac{K_{I I}^{2}}{\left(K_{I I c}\right)^{2}}=1
$$

what should replace the ultimate value of eq.(3.1)

This last equation further shows that fracture mechanics with constant stress intensity:

$$
K_{I c}=\sigma_{y} \sqrt{\pi c_{c}}=\sigma_{t} \sqrt{\pi r_{0} / 2}
$$


only applies when $r_{0}$ and $\sigma_{t}$, are constant. Thus $\sigma_{t}$ represents the cohesion strength of the crack tip crazes and $r_{0}$ is the invariant radius of the constant dimensions of the, "fracture process zone" (based on the material inhomogeneity structure of the craze, at the crack tip).

The, in [11], [12], chosen stress boundary definition: $K_{I}=\lim _{r \rightarrow 0}\left(\sigma_{22} \cdot(2 \pi r)^{1 / 2}\right)$ is arbitrary and is trivial, because the product $\left(\sigma_{22} \cdot(2 \pi r)^{1 / 2}\right)$ is independent of $\mathrm{r}$.

Hydrostatic tension, made possible by stress equalizing stress spreading effect in wood thus is shown to be possible in the fracture process zone and explains the hydrostatic stress effect for tension. It is probable that hydrostatic stress is generally caused by the stress spreading mechanism, in all materials, as is visual by the necked cross section, at flow, of a ductile iron rod.

\section{References:}

Outer, by [4], there are no recent developments of fundamental wood fracture mechanics theory. The last related hype, regarding "Fictitious crack models", ended, without discussion, certainly also due to the, in [15] given proof, of the not possible existence of strain softening.

[1] van der Put TACM (1982) A general failure criterion for wood, Proceed. 15th CIB-lUFRO Timber Engineering Group Meeting, Boras, Sweden, May 1982 or: Techn. Rep. 4-82-5 HA-14, Stevinlab. TU Delft, NL Feb. 1982.

[2] Hemmer, K. (1985) Versagensarten des Holzes der Weisstanne unter mehrassige Beanspruchung, Dissertation, Karlsruhe.

[3] van der Put TACM (2009) A continuum failure criterion applicable to wood, J Wood Sci (2009) 55:315-322.

[4] van der Put TACM (2017) A new fracture mechanics theory of wood, Extended second edition, Nova Science Publishers, New York

[5] Wu EM, (1963) Application of fracture mechanics to anisotropic plates, ASME J. Appl. Mech. Series E, 34 4, Dec. 1967, pp. 967-974..

[6] Mall S, Murphy JF, Shottafer JE, (1983) Criterion for Mixed Mode Fracture in Wood, J. Eng. Mech. 109(3) 680-690, June 1983.

[7] Stevenson AC (1945) Complex potentials in two-dimensional elasticity, Proc. Roy. Soc. London, A184, 129-79.

[8] Smith I, Landis E, Gong M, (2003) Fracture and Fatigue in Wood, J. Wiley \& Sns., Chichester, West Sussex, England

[9] Anderson TL (2005) Fracture Mechanics, Fundamentals and Applications 3rd edition, CRC Press Taylor \& Francis Group, Boca Raton, 2005

[10] Valentin GH., Boström l PJ. Gustafsson A, Ranta-Maunus, Gowda S, (1991) Application of fracture mechanics to timber structures RILEM state of the art report, Espoo, 1991 Technical Research Centre of Finland, Research Notes 1262.

[11] Schreurs PJG, (2012) Fracture Mechanics, Lecture notes course 4A780, Eindhoven University NL, concept version.

[12] Paris PC, Sih GC. (1965) Stress analysis of cracks. Symposium on Fracture Toughness Testing and its applications. Philadelphia PA, American society for Testing and materials, ASTM Special Technical Publication STP 381, 1965, Pp 30-80.

[13] van der Put TACM (2008) Derivation of the bearing strength perpendicular to the grain of locally loaded timber blocks Holz Roh Werkst (2008) 66: 409-417

[14] van der Put, TACM (1989) Deformation and damage processes in wood, Delft University press, 1989, Delft, NL

[15] van der Put, TACM (2015) The Myth of Softening behavior of the Cohesive Zone Model. Exact derivation of yield drop behavior of wood ISSN (e): 2250 - 3005 Vol 05 Issue 11 Nov 2015. Int J Comput Eng Res (IJCER) 